\title{
ON NORMAL TENSOR FUNCTORS AND COSET DECOMPOSITIONS FOR FUSION CATEGORIES
}

\author{
A. BRUGUIÈRES AND SEBASTIAN BURCIU
}

\begin{abstract}
We introduce the notion of double cosets relative to two fusion subcategories of a fusion category. Given a tensor functor $F: \mathcal{C} \rightarrow \mathcal{D}$ between fusion categories, we introduce an equivalence relation $\approx^{F}$ on the set $\Lambda_{\mathcal{C}}$ of isomorphism classes of simple objects of $\mathcal{C}$, and when $F$ is dominant, an equivalence relation $\approx_{F}$ on $\Lambda_{\mathcal{D}}$. We show that the equivalent classes of $\approx^{F}$ are cosets. We also give a description of the image of $F$ when it is a normal tensor functor, and we show that $F$ is normal if and only if the images of $\approx^{F}$ equivalent elements of $\Lambda_{\mathcal{C}}$ are colinear. We study the situation where the composition of two tensor functors $F=F^{\prime} F^{\prime \prime}$ is normal, and we give a criterion of normality for $F^{\prime \prime}$, with an application to equivariantizations. Lastly, we introduce the radical of a fusion subcategory and compare it to its commutator in the case of a normal subcategory. We also give a description for the image of a normal tensor functor between any two fusion categories.
\end{abstract}

\section{INTRODUCTION}

In this paper we introduce the notion of double cosets in a fusion category relative to two fusion subcategories, which generalize double cosets in a group relative to two subgroups, and we use this notion to study tensor functors between fusion categories. Double cosets can be studied in terms of the corresponding regular elements in the Grothendieck ring of the fusion category.

We show that a tensor functor $F: \mathcal{C} \rightarrow \mathcal{D}$ between fusion categories gives rise to two equivalence relations: one, denoted by $\approx^{F}$, on the set of simple objects $\Lambda_{\mathcal{C}}$ of isomorphism classes of simple objets of $\mathcal{C}$, and another (if $F$ is dominant), denoted by $\approx_{F}$, on the set $\Lambda_{\mathcal{D}}$ of simple objects of $\mathcal{D}$. These equivalence relations are the categorical analogues of those introduced by Rieffel in [14] for the restriction functor attached to an extension of semisimple rings. We prove that the equivalence classes for $\approx^{F}$ are left (and right) cosets relative to a certain fusion subcategory of $\mathcal{C}$.

Date: June 21, 2018. 
In the special case of a normal tensor functor (a notion introduced in [3]); we give a description of images of objects under normal tensor functors $F: \mathcal{C} \rightarrow \mathcal{D}$. In particular, we show that normal functors are characterized by the fact that objects in the same equivalence class in $\Lambda_{\mathcal{C}}$ have colinear images in the Grothendieck ring of $\mathcal{D}$. A similar result holds for the equivalence classes in $\Lambda_{\mathcal{D}}$ when $F$ is dominant.

We study the situation when a composite of tensor functors $F=$ $F^{\prime} F^{\prime \prime}$ is normal; we show that if $F^{\prime \prime}$ is dominant and and $F$ normal then $F^{\prime}$ is also normal. We also give a criterion of normality for $F^{\prime \prime}$ in Theorem 5.1. As an illustration, we apply this result to equivariantizations. Denote by $\mathcal{C}^{G}$ the equivariantization of a fusion category $\mathcal{C}$ under the action of a finite group $G$ by tensor autoequivalences; it is again a fusion category under some reasonable hypotheses $(e . g$. over $\mathbb{C}$ ). We obtain that if $H$ is a subgroup of $G$, then the restriction functor $\mathcal{C}^{G} \rightarrow \mathcal{C}^{H}$ is normal if and only if $H$ is a normal subgroup of $G$, and in that case, $\mathcal{C}^{G}$ is equivalent to $\left(\mathcal{C}^{H}\right)^{G / H}$ for a certain action of $G / H$ on $\mathcal{C}^{H}$ by tensor automorphisms.

Lastly, by analogy with ring theory, we define the radical of a fusion subcategory of a fusion category $\mathcal{C}$. We show that the radical of a normal subcategory coincides with its commutator (defined in [11]). Recall that a normal subcategory $\mathcal{D}$ of a fusion category of $\mathcal{C}$ (as defined in [3]) is the kernel of a normal tensor functor $F: \mathcal{C} \rightarrow \mathcal{E}$ from $\mathcal{C}$ to another fusion category $\mathcal{E}$. The kernel of $F$ is the full subcategory of $\mathcal{C}$ consisting of all objects whose image by $F$ is a multiple of the unit object.

This paper is organized as follows. In the first section we recall known facts facts about fusion categories and tensor functors which we need. In Section 2 we define double cosets in a fusion category $\mathcal{C}$ relative to two fusion subcategories $\mathcal{D}$ and $\mathcal{E}$, and give the corresponding decomposition of $\mathcal{C}$ into indecomposable bimodule categories, and also the corresponding decomposition of the regular virtual object of $\mathcal{C}$ in the Grothendieck ring. In Section 3 , we introduce and study two equivalence relations $\approx^{F}$ and $\approx_{F}$ attached to a tensor functor $F$. In fact $\approx^{F}$ is a coset equivalence relation (Proposition 3.3). In Section 4 we give a description of the image of simple objects under a normal tensor functor, and a new characterization of normal functors. In Section 5 , we study the situation where the composition of two tensor functors $F=F^{\prime} F^{\prime \prime}$ is normal, and in particular, a criterion of normality for $F^{\prime \prime}$, with an application to equivariantizations. In Section [6] we introduce 
the radical of a fusion subcategory, and compare it to its commutator in the case of a normal subcategory.

\section{Preliminaries}

In this section we recall the basic facts on fusion categories and tensor functors that are needed in this paper.

1.1. Fusion categories. Let $\mathbb{k}$ be a field. A fusion category over $\mathbb{k}$ is a $\mathbb{k}$-linear semisimple monoidal rigid category $\mathcal{C}$ with finitely many isomorphism classes of simple objects, finite dimensional homomorphism spaces, such that each simple object $S$ is scalar (that is, $\operatorname{End}(S)=\mathbb{k}$ ) and the unit object $\mathbf{1}$ of $\mathcal{C}$ is simple.

We refer the reader to 9 for more details on fusion categories. A fusion subcategory of a fusion category $\mathcal{C}$ is a full replete monoidal subcategory of $\mathcal{C}$ which is also a fusion category.

In a fusion category $\mathcal{C}$, the left and right duals ${ }^{*} X$ and $X^{*}$ of an object $X$ are isomorphic (but it is still not known whether there always exists a sovereign structure, that is a natural monoidal isomorphism between the two duals).

Denote by $\Lambda_{\mathcal{C}}$ the set of isomorphism classes of objects of $\mathcal{C}$, and by $\operatorname{Inv}(\mathcal{C}) \subset \Lambda_{\mathcal{C}}$ the set of isomorphism classes of invertible objects of $\mathcal{C}$ (which is a group for the tensor product). If $X$ is an object of a fusion category $\mathcal{C}$, we denote by $\langle X\rangle$ the smallest fusion subcategory of $\mathcal{C}$ containing $X$.

An object of $\mathcal{C}$ is trivial if it belongs to $\langle\mathbf{1}\rangle$, that is, if it is isomorphic to $\mathbf{1}^{n}$ for some $n \in \mathbb{N}$.

The Grothendieck ring $K_{0}(\mathcal{C})$ of $\mathcal{C}$ is the free $\mathbb{Z}$-module

$$
K_{0}(\mathcal{C})=\bigoplus_{X \in \Lambda_{\mathcal{C}}} \mathbb{Z}[X]
$$

equipped with the product defined by the tensor product of $\mathcal{C}$ (if $X$ is an (isomorphism class of) simple object of $\mathcal{C}$, we denote by $[X]$ the corresponding basis element of $K_{0}(\mathcal{C})$ ). A virtual object of $\mathcal{C}$ is an element of the ring $K_{0}(\mathcal{C})_{\mathbb{C}}=K_{0}(\mathcal{C}) \otimes_{\mathbb{Z}} \mathbb{C}$, that is, a formal complex linear combination of elements of $\Lambda_{\mathcal{C}}$.

Duality induces an involution $?^{*}$ on $K_{0}(\mathcal{C})$ given by $[X]^{*}:=\left[X^{*}\right]$.

For a simple object $X$ let $\mathrm{FPdim}(X)$ denote the Frobenius-Perron dimension of $X$, that is, the Frobenius-Perron eigenvalue of the left multiplication by $[X]$ on the Grothendieck ring $K_{0}(\mathcal{C})$. It is a positive real algebraic number. The Frobenius-Perron dimension extends linearly to an algebra morphism FPdim $: K_{0}(\mathcal{C})_{\mathbb{C}} \rightarrow \mathbb{C}$. 
The regular virtual object of $\mathcal{C}$ is the virtual object:

$$
\mathrm{R}_{\mathcal{C}}=\sum_{X \in \Lambda_{\mathcal{C}}} \operatorname{FPdim}(X)[X]
$$

and the Frobenius-Perron dimension $\operatorname{FPdim}(\mathcal{C})$ of $\mathcal{C}$ is the FrobeniusPerron dimension of $R_{\mathcal{C}}$, that is:

$$
\operatorname{FPdim}(\mathcal{C}):=\sum_{X \in \Lambda_{\mathcal{C}}} \operatorname{FPdim}(X)^{2}
$$

The regular virtual object satisfies

$$
x \mathrm{R}_{\mathcal{C}}=\operatorname{FPdim}(x) \mathrm{R}_{\mathcal{c}}=\mathrm{R}_{\mathcal{c}} x
$$

for any $x \in K_{0}(\mathcal{C})_{\mathbb{C}}($ see $[9])$. In particular $\mathrm{R}_{\mathcal{C}}^{2}=\operatorname{FPdim}(\mathcal{C}) \mathrm{R}_{\mathcal{C}}$.

Denote by $m_{\mathcal{C}}$ the $\mathbb{Z}$-bilinear form on the Grothendieck ring $K_{0}(\mathcal{C})$ defined by

$$
m_{\mathcal{C}}([X],[Y])=\operatorname{dim}_{\mathbb{k}} \operatorname{Hom}(X, Y) \text { for } X, Y \text { simple objects of } \mathcal{C} .
$$

The bilinear form $m_{\mathcal{C}}$ has the following properties:

(1) symmetry: $m_{\mathcal{C}}(x, y)=m_{\mathcal{C}}(y, x)$;

(2) adjunction property: $m_{\mathcal{C}}(x, y z)=m_{\mathcal{C}}\left(y^{*} x, z\right)=m_{\mathcal{C}}\left(x z^{*}, y\right)$

for $x, y, z \in K_{0}(\mathcal{C})$.

We also denote by $m_{\mathcal{C}}$ the extension of this form to a $\mathbb{C}$-bilinear form on the ring $K_{0}(\mathcal{C})_{\mathbb{C}}$ of virtual objects of $\mathcal{C}$.

Given any subset $A \subset \Lambda_{\mathcal{C}}$, we denote by $\mathrm{R}_{A}$ the virtual object

$$
\mathrm{R}_{A}=\sum_{X \in A} \operatorname{FPdim}(X)[X] \in K_{0}(\mathcal{C})_{\mathbb{C}}
$$

In particular $\mathrm{R}_{\mathcal{C}}=\mathrm{R}_{\Lambda_{\mathcal{C}}}$.

Given a $\mathbb{k}$-linear functor $G: \mathcal{C} \rightarrow \mathcal{C}^{\prime}$ between fusion categories, we

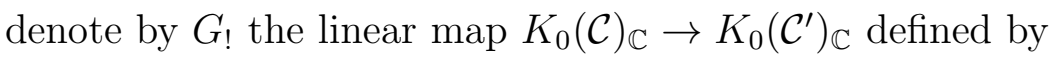

$$
G_{!}[X]=\sum_{Y \in \Lambda_{\mathcal{C}^{\prime}}} m_{\mathcal{C}^{\prime}}(Y, G(X))[Y] .
$$

1.2. Tensor functors between fusion categories. A tensor functor $F: \mathcal{C} \rightarrow \mathcal{D}$ between two fusion categories $\mathcal{C}$ and $\mathcal{D}$ over a field $\mathbb{k}$ is a strong monoidal $\mathbb{k}$-linear functor $F: \mathcal{C} \rightarrow \mathcal{D}$.

Let $F: \mathcal{C} \rightarrow \mathcal{D}$ be a tensor functor between fusion categories. The kernel of $F$ is the fusion subcategory $\mathfrak{K e r}_{F} \subset \mathcal{C}$ consisting of all objects $X$ of $\mathcal{C}$ such that $F(X)$ is trivial. It is endowed with a canonical fiber functor $\omega: \mathfrak{K e r}_{F} \rightarrow \operatorname{Vec}_{\mathbb{k}}, X \mapsto \operatorname{Hom}_{\mathcal{D}}(\mathbf{1}, F(X))$. Hence, by Tannaka reconstruction, one obtains a finite dimensional Hopf algebra $H$ over $\mathbb{k}$ such that $\mathfrak{K e r}_{F} \simeq \operatorname{comod}-H$ (see [3] for more details). 
We say that $F$ is dominant if for $Y \in \Lambda_{\mathcal{D}}$ there exists $X \in \Lambda_{\mathcal{C}}$ such that $Y$ is a factor of $F(X)$. The dominant image of $F$ is the fusion subcategory $\mathcal{D}^{\prime} \subset \mathcal{D}$ generated by the image of $F$; and $F$ is dominant if and only if $\mathcal{D}^{\prime}=\mathcal{D}$. Note that $F$ can always be factorized as a dominant functor $\mathcal{C} \rightarrow \mathcal{D}^{\prime}$, followed by the inclusion $\mathcal{D}^{\prime} \hookrightarrow \mathcal{D}$.

We say that $F$ is normal (a notion introduced in [3]) if for any $X \in \Lambda_{\mathcal{C}}$ such that $F(X)$ contains the unit object $\mathbf{1}, F(X)$ is trivial (that is, isomorphic to $\mathbf{1}^{n}$ for $n \in \mathbb{N}$ ). In that case, the Hopf algebra $H$ is called the induced Hopf algebra of $F$.

Note that a tensor functor between fusion categories admits a left adjoint and a right adjoint. Let us denote by $R$ the right adjoint of $F$. Then $F$ is dominant if and only if $R$ is faithful, and $F$ is normal if and only if $F R(\mathbf{1})$ is trivial. The object $A=R(\mathbf{1})$ is an algebra in $\mathcal{C}$, called the induced algebra of $F$. Moreover, we have canonical natural isomorphisms for $X$ in $\mathcal{C}, Y$ in $\mathcal{D}$ :

$$
X \otimes R(Y) \stackrel{\sim}{\longrightarrow} R(F X \otimes Y) \text { and } R(Y) \otimes X \stackrel{\sim}{\longrightarrow} R(Y \otimes F X),
$$

and in particular (taking $Y=1$ ), we have isomorphisms $A \otimes X \stackrel{\sim}{\longrightarrow}$ $R F(X) \stackrel{\sim}{\longrightarrow} X \otimes A$. The natural transformation $\sigma_{X}: A \otimes X \stackrel{\sim}{\longrightarrow} X \otimes A$ so defined is a half-braiding, and $\mathbb{A}=(A, \sigma)$ is a commutative algebra in the categorical center $\mathcal{Z}(\mathcal{C})$ of $\mathcal{C}$ called the induced central algebra of $F$. See [1] for a detailed account in a more general setting.

A fusion subcategory $\mathcal{D} \subset \mathcal{C}$ is normal (in the sense of [3]) if there exists a normal tensor functor $F: \mathcal{C} \rightarrow \mathcal{E}$ between fusion categories such that $\mathcal{D}=\mathfrak{K e r}_{F}$.

1.3. Exact sequences of fusion categories. The notion of an exact sequence of tensor categories, introduced in [3] for tensor (not necessarily semisimple) categories, generalizes the classical notion of an exact sequence of groups and the notion of an exact sequence of Hopf algebras due to Schneider. Here, we restrict our attention to fusion categories.

An exact sequence of fusion categories is a diagram of tensor functors between fusion categories

$$
\text { (E) } \quad \mathcal{C}^{\prime} \stackrel{i}{\longrightarrow} \mathcal{C} \stackrel{F}{\longrightarrow} \mathcal{C}^{\prime \prime}
$$

such that:

(1) $i$ is fully faithful;

(2) $F$ is normal and dominant;

(3) the essential image of $i$ is $\mathfrak{K e r}_{F}$.

We will use the following result on exact sequences (multiplicativity of Frobenius-Perron dimensions): 
Proposition 1.1 ([3], Proposition 3.10). Consider a diagram of tensor functors between fusion categories

$$
\text { (E) } \quad \mathcal{C}^{\prime} \stackrel{i}{\longrightarrow} \mathcal{C} \stackrel{F}{\longrightarrow} \mathcal{C}^{\prime \prime}
$$

such that $i$ is fully faithful, $F$ is dominant, and $i\left(\mathcal{C}^{\prime}\right) \subset \mathfrak{K e r}_{F}$. Then

$$
\operatorname{FPdim}(\mathcal{C}) \geq \operatorname{FPdim}\left(\mathcal{C}^{\prime}\right) \operatorname{FPdim}\left(\mathcal{C}^{\prime \prime}\right)
$$

and equality holds if and only if $(E)$ is an exact sequence.

Interesting examples of exact sequences are provided by equivariantizations. Let $G$ be a finite group, and $\mathcal{C}$ be a fusion category. An action of $G$ on $\mathcal{C}$ by tensor autoequivalences is a strong monoidal functor $\rho: G \rightarrow \operatorname{Aut}_{\otimes}(\mathcal{C})$.

Given such an action, let $\mathcal{C}^{G}$ be the equivariantization of $\mathcal{C}$ under the action of $G$. Recall that the objects of $\mathcal{C}^{G}$ are pairs $\left(X, \underline{r}=\left(r_{g}\right)_{g \in G}\right)$, where $X$ is an object of $\mathcal{C}$, and the $r_{g}$ 's are isomorphisms $r_{g}: \rho(g)(X) \rightarrow$ $X$ satisfying certain compatibilities (see [12] for more details). The equivariantization $\mathcal{C}^{G}$ is a tensor (in general, not fusion) category, and the forgetful functor $U_{G}: \mathcal{C}^{G} \rightarrow \mathcal{C},(X, \underline{r}) \mapsto X$ is a tensor functor.

The tensor category $\mathcal{C}^{G}$ is a fusion category provided $\mathbb{k}$ is algebraically closed and its characteristic does not divide to the order of $G$.I $\mathrm{n}$ that case, a detailed description of the simple objects of $\mathcal{C}^{G}$ is provided in [6].

As shown in [3], in that situation $F$ is normal dominant, $\mathfrak{K e r}_{F}$ can be identified with $\operatorname{Rep}(G)$, and we have therefore an exact sequence of fusion categories:

$$
\operatorname{Rep}(G) \longrightarrow \mathcal{C}^{G} \longrightarrow \mathcal{C} .
$$

We say that a tensor functor $F: \mathcal{C} \rightarrow \mathcal{D}$ between fusion categories is an equivariantization if there exists a finite group $G$ acting on $\mathcal{D}$ by tensor autoequivalences, and a tensor equivalence $K: \mathcal{C} \rightarrow \mathcal{D}^{G}$ such that $F=U_{G} K$.

In [3, 2], several criteria are given for a tensor functor to be an equivariantization, notably in terms of central exact sequences.

An exact sequence $(E) \quad \mathcal{C}^{\prime} \longrightarrow \mathcal{C} \stackrel{F}{\longrightarrow} \mathcal{C}^{\prime \prime}$ of fusion categories is central if, denoting by $\mathbb{A}=(A, \sigma)$ the induced central algebra of $F$, the forgetful functor $\mathcal{Z}(\mathcal{C}) \rightarrow \mathcal{C}$ induces an equivalence of categories $\langle\mathbb{A}\rangle \rightarrow\langle A\rangle$.

In the fusion case, we have the following

Theorem 1.2. Let $(E) \quad \mathcal{C}^{\prime} \longrightarrow \mathcal{C} \stackrel{F}{\longrightarrow} \mathcal{C}^{\prime \prime}$ be an exact sequence of fusion categories over a field $\mathbb{k}$. The following assertions are equivalent: 
(i) The functor $F$ is an equivariantization for an action of a finite group $G$ acting on $\mathcal{C}^{\prime \prime}$ by tensor autoequivalences;

(ii) $(E)$ is a central exact sequence and its induced Hopf algebra is split semisimple.

When these assertions hold, $H$ is commutative and $G=\operatorname{Spec}(H)$.

Proof. This results directly from [2], Proposition 3.2, combined with [2], Theorem 3.6.

1.4. Module and bimodule categories. The notion of a module category is a categorification of the notion of a module over a ring.

If $\mathcal{C}$ is a monoidal category, a left module category over $\mathcal{C}$ is a plain category $\mathcal{M}$ endowed with a strong monoidal functor $\rho: \mathcal{C} \rightarrow \underline{\operatorname{End}}(\mathcal{M})$. In other words, it is a category $\mathcal{M}$ with a action bifunctor $\otimes: \mathcal{C} \times \mathcal{M} \rightarrow$ $\mathcal{M}$ with an associativity constraint $a_{U, V, M}: U \otimes(V \otimes M) \cong(U \otimes V) \otimes M$ and a unit constraint $1 \otimes M \cong M$ satisfying a pentagon and a triangle coherence axiom, see [13].

We will restrict ourselves to the case where $\mathcal{C}$ is a fusion category over a field $\mathbb{k}, \mathcal{M}$ is a $\mathbb{k}$-linear semisimple abelian category, and the action bifunctor is $\mathbb{k}$ - linear in each variable. Such a module category is indecomposable if $\mathcal{M}$ is not a direct sum of two nontrivial module subcategories.

Let $\mathcal{C}$ and $\mathcal{D}$ be fusion category. Denote by $\otimes$ the tensor product of abelian categories introduced in [7]. A $(\mathcal{C}, \mathcal{D})$-bimodule category is a left $\mathcal{C} \otimes \mathcal{D}^{\text {rev }}$-module category. Here $\mathcal{D}^{\text {rev }}$ denotes $\mathcal{D}$ with opposite monoidal structure.

\section{Coset DeCompositions For FUsion CATEGories}

2.1. Double coset decomposition for fusion categories. Let $\mathcal{C}$ be a fusion category and $\mathcal{D}, \mathcal{E}$ be two fusion subcategories of $\mathcal{C}$. Define a relation $\sim$ on $\Lambda_{\mathcal{C}}$ by

$$
X \sim Y \Longleftrightarrow \exists D \in \Lambda_{\mathcal{D}}, E \in \Lambda_{\mathcal{E}} \mid Y \text { is a factor of } D \otimes X \otimes E .
$$

Observe that we have $X \sim Y$ if and only if $m_{\mathcal{C}}\left(X, \mathrm{R}_{\mathcal{D}} Y \mathrm{R}_{\mathcal{E}}\right)>0$, where $\mathrm{R}_{\mathcal{D}}$ and $\mathrm{R}_{\mathcal{E}}$ denote the regular virtual objects of $\mathcal{D}$ and $\mathcal{E}$.

Lemma 2.1. The relation $\sim$ is an equivalence relation on $\Lambda_{\mathcal{C}}$.

Proof. Let $X \in \Lambda_{\mathcal{C}}$. Since the unit object of $\mathcal{C}$ belongs to $\mathcal{D}$ and $\mathcal{E}$, we have $X \sim X$, which proves the reflexivity of $\sim$. Let $X, Y \in \Lambda_{\mathcal{C}}$ such that $X \sim Y$. There exist $D \in \Lambda_{\mathcal{D}}, E \in \Lambda_{\mathcal{E}}$ such that $Y$ is a factor of $D \otimes X \otimes E$, that is, $m_{\mathcal{C}}(Y, D \otimes Y \otimes E)>0$. By symmetry and adjunction, $m_{\mathcal{C}}\left(X, D^{*} \otimes Y \otimes E^{*}\right)=m_{\mathcal{C}}\left(D^{*} \otimes Y \otimes E^{*}, X\right)=m_{\mathcal{C}}(Y, D \otimes X \otimes E)>0$, so $X$ is a factor of $D^{*} \otimes Y \otimes E^{*}$, and, $\mathcal{D}$ and $\mathcal{E}$ being stable by duality, 
that implies $Y \sim X$. So, $\sim$ is symmetric. Lastly, let $X, Y, Z \in \Lambda_{\mathcal{C}}$ and assume $X \sim Y$ and $Y \sim Z$. There exist $D, D^{\prime} \in \Lambda_{\mathcal{D}}, E, E^{\prime} \in \Lambda_{\mathcal{E}}$ such that $Y$ is a factor of $D \otimes X \otimes E$ and $Z$ is a factor of $D^{\prime} \otimes Y \otimes E^{\prime}$. Therefore, $Z$ is a factor of $D^{\prime} \otimes D \otimes E \otimes E^{\prime}$. Now we have $D^{\prime} \otimes D=$ $D_{1} \oplus \cdots \oplus D_{k}$ and $E \otimes E^{\prime}=E_{1} \oplus \cdots \oplus E_{l}$, where the $D_{i}$ 's and the $E_{j}$ 's are (isomorphy classes of) simple objects of $\mathcal{D}$ and $\mathcal{E}$ respectively, and $Z$, being simple, is a factor of one of the $D_{i} \otimes X \otimes E_{j}$ 's, which shows that $X \sim Z$. Therefore $\sim$ is transitive.

From now on, the equivalence relation $\sim$ will be denoted by $r_{\mathcal{D}, \mathcal{E}}^{\mathcal{C}}$. Its equivalence classes are called $\mathcal{D}-\mathcal{E}$ double cosets. If $B$ is a $\mathcal{D}-\mathcal{E}$ double coset, the full subcategory $\mathcal{B} \subset \mathcal{C}$ whose objects are finite direct sums of simple objects belonging to $B$ is called a $\mathcal{D}-\mathcal{E}$ double coset subcategory.

Example 2.2. If $G$ is a finite group, denote by $\mathcal{C}=\operatorname{Vec}_{G}$ the fusion category of finite-dimensional $G$-graded vector spaces. For any two subgroups $H, L \subset G$ let $\mathcal{D}:=\operatorname{Vec}_{H}$ and $\mathcal{E}:=\operatorname{Vec}_{L}$. Then $\mathcal{D}-\mathcal{E}$ double cosets in $\mathcal{C}$ are just double cosets $H \backslash G / L$.

Remark 2.3. The equivalence relation $r_{\mathcal{D}, \mathcal{E}}^{\mathcal{C}}$, in the special case $\mathcal{D}=$ Vec, appears in [8, page 26] in the study of the centralizer of a fusion subcategory of a braided fusion category.

Proposition 2.4. Let $\mathcal{C}$ be a fusion category and $\mathcal{D}, \mathcal{E}$ be two fusion subcategories $\mathcal{C}$. Let $\mathcal{B}_{1}, \ldots, \mathcal{B}_{l}$ be the $\mathcal{D}-\mathcal{E}$ double coset subcategories of $\mathcal{C}$. Then we have a decomposition of $\mathcal{C}$ as sum of indecomposable $\mathcal{D}-\mathcal{E}$ bimodule categories

$$
\mathcal{C}=\bigoplus_{i=1}^{l} \mathcal{B}_{i}
$$

Proof. We have $\mathcal{C}=\bigoplus \mathcal{B}_{i}$ as $\mathbb{k}$-linear categories because double cosets form a partition of $\Lambda_{\mathcal{C}}$. The category $\mathcal{C}$ is a $\mathcal{D}$ - $\mathcal{E}$ module category, $\mathcal{D}$ acting by tensoring on the left, and $\mathcal{E}$ on the right. The definition of $r_{\mathcal{D}, \mathcal{E}}^{\mathcal{C}}$ ensures that the $\mathcal{B}_{i}$ 's are indecomposable sub-bimodule categories of $\mathcal{C}$.

Remark 2.5. It follows from [9, Remark 8.17] that both $\frac{\operatorname{FPdim}\left(\mathcal{B}_{i}\right)}{\operatorname{FPdim}(\mathcal{D})}$ and $\frac{\operatorname{FPdim}\left(\mathcal{B}_{i}\right)}{\operatorname{FPdim}(\mathcal{E})}$ are algebraic integers.

2.2. The regular virtual object of a double coset. Let $\mathcal{C}$ be a fusion category, and $\mathcal{D}, \mathcal{E} \subset \mathcal{C}$ be two fusion subcategories. If $B$ is a $\mathcal{D}-E$ double coset, set

$$
K_{0}(B)_{\mathbb{C}}=\oplus_{[X] \in B} \mathbb{C}[X] \subset K_{0}(\mathcal{C})_{\mathbb{C}}
$$


and recall the notation

$$
\mathrm{R}_{B}=\sum_{[X] \in B} \operatorname{FPdim}(X)[X] \in K_{0}(B)_{\mathbb{C}} .
$$

Let $B_{1}, \ldots, B_{l}$ be the list of all double cosets, so that we have

$$
K_{0}(\mathcal{C})_{\mathbb{C}}=\bigoplus_{i=1}^{l} K_{0}\left(B_{i}\right)_{\mathbb{C}} \quad \text { and } \quad \mathrm{R}_{\mathcal{C}}=\sum_{i=1}^{l} \mathrm{R}_{B_{i}}
$$

and denote by $T$ the operator on $K_{0}(\mathcal{C})_{\mathbb{C}}$ defined by $T x=\mathrm{R}_{\mathcal{D}} x \mathrm{R}_{\mathcal{E}}$.

Recall from [10 that a matrix $A \in M_{n}(\mathbb{C})$ is called indecomposable if the set $I=\{1,2, \cdots, n\}$ cannot be written as a disjoint union $I=J_{1} \cup J_{2}$ with $J_{1} \neq \emptyset$ and $J_{2} \neq \emptyset$, in such a way that $a_{u v}=0$ whenever $u \in J_{1}$ and $v \in J_{2}$.

Proposition 2.6. With the above notations, for each double coset $B$ the space $K_{0}(B)_{\mathbb{C}}$ is stable under the operator $T$. Denoting by $T_{B}$ the restriction of $T$ to $K_{0}(B)_{\mathbb{C}}$, the matrix of $T_{B}$ in the basis $B$ has real non-negative entries and is indecomposable. Its Frobenius-Perron eigenvalue is $\mathrm{FP} \operatorname{dim}(\mathcal{D}) \mathrm{FP} \operatorname{dim}(\mathcal{E})$ and the corresponding eigenspace is the line generated by $\mathrm{R}_{B}$.

Proof. The stability of $K_{0}(B)_{\mathbb{C}}$ under $T$, and the fact that the matrix of $T$ in the basis $B$ is indecomposable, are immediate consequences of the definition of the equivalence relation $r_{\mathcal{D}, \mathcal{E}}^{\mathcal{C}}$. The entries of the matrix are real non-negative because the coefficients of $R_{\mathcal{D}}$ and $R_{\mathcal{E}}$ are real nonnegative. Thus, the Frobenius-Perron theorem (see [10]) applies to the matrix of $T_{B}$ : its spectral radius $\lambda$ is an eigenvalue (the FrobeniusPerron eigenvalue), and the corresponding eigenspace has dimension 1 and is generated by a vector $x$ with positive coordinates. In particular $\operatorname{FPdim}(x)$ is positive.

We have $T_{B} x=\mathrm{R}_{\mathcal{D}} x \mathrm{R}_{\mathcal{\varepsilon}}=\lambda x$, and, taking Frobenius-Perron dimensions we obtain $\mathrm{FPdim}(\mathcal{D}) \mathrm{FPdim}(x) \mathrm{FPdim}(E)=\lambda \mathrm{FPdim}(x)$, hence $\lambda=\mathrm{FPdim}(\mathcal{D}) \mathrm{FPdim}(E)$.

On the other hand, we have $T \mathrm{R}_{\mathcal{C}}=\mathrm{R}_{\mathcal{D}} \mathrm{R}_{\mathcal{C}} \mathrm{R}_{\mathcal{D}}=\operatorname{FPdim}(\mathcal{D}) \operatorname{FPdim}(\mathcal{E}) \mathrm{R}_{\mathcal{C}}$, and since $\mathrm{R}_{\mathcal{C}}=\sum_{i=1}^{l} \mathrm{R}_{B_{i}}$, we see that $\mathrm{R}_{B_{i}}$ 's are eigenvectors of $T$ for the eigenvalue $\lambda$. In particular $v=\mathrm{R}_{B}$ up to a positive scalar.

Corollary 2.7. With the above notations, if $X \in B$ then

$$
\mathrm{R}_{\mathcal{D}}[X] \mathrm{R}_{\mathcal{E}}=\operatorname{FPdim}(X) \frac{\operatorname{FPdim}(\mathcal{D}) \operatorname{FPdim}(\mathcal{E})}{\operatorname{FPdim}\left(\mathrm{R}_{B}\right)} \mathrm{R}_{B}
$$


Proof. Let $y=\mathrm{R}_{\mathcal{D}}[X] \mathrm{R}_{\mathcal{E}} \in K_{0}(B)_{\mathbb{C}}$. We have $T_{B} y=\mathrm{R}_{\mathcal{D}}^{2}[X] \mathrm{R}_{\mathcal{E}}^{2}=$ $\operatorname{FPdim}(\mathcal{D}) \mathrm{FPdim}(\mathcal{E}) y$, so by Proposition 2.6, $y=t \mathrm{R}_{B}$ for some scalar $t$. We have $\mathrm{FPdim}(y)=\mathrm{FPdim}(\mathcal{D}) \mathrm{FPdim}(E) \mathrm{FPdim}(X)=t \mathrm{FPdim}\left(\mathrm{R}_{B}\right)$, hence the value of $t$. This proves the corollary.

Example 2.8. Let $H$ be a finite dimensional semisimple Hopf algebra over an algebraically closed field $\mathbb{k}$ of characteristic 0 . Then $H$ is also cosemisimple. Let $K$ and $L$ be two Hopf subalgebras of $H$. Then the category $\mathcal{C}=\operatorname{comod} H$ of finite dimensional right $H$-comodules is a fusion category, and $\mathcal{D}=\operatorname{comod} K$ and $\mathcal{E}=\operatorname{comod} L$ are fusion subcategories of $\mathcal{C}$. In this case, the equivalence relation $r_{\mathcal{D}, \mathcal{E}}^{\mathcal{C}}$ coincides with the equivalence relation $r_{K, L}^{H}$ on the set of simple right comodules of $H^{*}$ introduced in [4].

Remark 2.9. The theory above allows one to extend the notion of double cosets for Hopf subalgebras from [4] to double cosets for coquasi Hopf subalgebras of a given co-quasi Hopf algebra.

We denote by $r_{\mathcal{E}}^{\mathcal{C}, r}$ (resp. $r_{\mathcal{E}}^{\mathcal{C}, l}$ ) the equivalence relation $r_{\text {Vec, } \mathcal{E}}^{\mathcal{C}}$ (resp. $\left.r_{\mathcal{E}, \text { Vec }}^{\mathcal{C}}\right)$. Its equivalence classes are called the left (resp. right) cosets of $\mathcal{E}$ in $\mathcal{C}$.

Example 2.10. Let $\mathcal{C}$ be a fusion category, and let

$$
\mathcal{C}=\bigoplus_{g \in G} \mathcal{C}_{g}
$$

be a $G$-graduation of $\mathcal{C}$, for some finite group $G$. Assume that the graduation is faithful, that is, all $\mathcal{C}_{g}$ 's are non-zero. Then each $\mathcal{C}_{g}$ is both a left and a right coset subcategory with respect to $\mathcal{C}_{1}$.

Indeed, let $X, Y \in \Lambda_{\mathcal{C}}$. If $X$ and $Y$ belong to the same left coset, there exists $Z \in \Lambda_{\mathcal{C}_{1}}$ such that $Y$ is a factor of $Z \otimes X$, so $X$ and $Y$ have same degree. Conversely, if $X$ and $Y$ have same degree, then $Y$ is a factor of $Y \otimes X^{*} \otimes X$, with $Y \otimes X^{*}$ of degree 1 , so $X$ and $Y$ belongs to same the left coset. This shows that the $\mathcal{C}_{g}$ 's are left coset subcategories. Similarly, they are right coset subcategories.

In particular, the fusion subcategories of $\mathcal{C}$ containing $\mathcal{C}_{1}$ are in bijection with the subgroups of $G$.

\section{Two EqUiVAlenCE RELATIONS ASSOCIATED With A TENSOR FUNCTOR BETWEEN FUSION CATEGORIES}

Let $F: \mathcal{C} \rightarrow \mathcal{D}$ be a tensor functor between fusion categories, and denote by $R$ its right adjoint. 
For $X \in \Lambda_{\mathcal{C}}$ set

$$
X^{F}=\left\{[Y] \in \Lambda_{\mathcal{D}} \mid Y \text { is a factor of } F(X)\right\}
$$

and for $Y \in \Lambda_{\mathcal{D}}$, set

$$
Y_{F}=\left\{[X] \in \Lambda_{\mathcal{C}} \mid Y \text { is a factor of } F(X)\right\} .
$$

We have $X \in Y_{F} \Longleftrightarrow Y \in X^{F} \Longleftrightarrow X$ is a factor of $R(Y)$.

Remark 3.1. Note that $F$ is dominant if and only if $\Lambda_{\mathcal{C}}^{F}=\Lambda_{\mathcal{D}}$.

3.1. An equivalence relation on $\Lambda_{\mathcal{C}}$ induced by $F$. Define a relation $\sim^{F}$ on $\Lambda_{\mathcal{C}}$ by $X \sim^{F} X^{\prime} \Longleftrightarrow X^{F} \cap X^{\prime F} \neq \emptyset$. The relation $\sim^{F}$ is clearly reflexive and symmetric. However, it is not transitive in general:

Example 3.2. Denote by $S_{n}$ the $n$-th symmetric group. The standard inclusion $S_{n} \subset S_{n+1}$ defines by restriction a tensor functor from the category of $\mathbb{C} S_{n+1}$-modules to the category of $\mathbb{C} S_{n}$-modules. It follows from Theorem 6.19 of [5] that $\sim^{F}$ is not an equivalence relation.

Denote by $\approx^{F}$ the transitive closure of $\sim^{F}$, which is an equivalence relation on $\Lambda_{\mathcal{C}}$.

Proposition 3.3. Let $F: \mathcal{C} \rightarrow \mathcal{D}$ a tensor functor between fusion categories, with right adjoint $R$. Let $\mathcal{A}=\langle R(\mathbf{1})\rangle$ be the fusion subcategory of $\mathcal{C}$ generated by $R(\mathbf{1})$.

Then $\approx^{F}=r_{\mathcal{A}}^{C, r}=r_{\mathcal{A}}^{C, l}$. In other words, the equivalence classes of $\approx^{F}$ are the left cosets with respect to $\mathcal{A}$, which coincide with the right cosets with respect to $\mathcal{A}$.

Proof. Let $A=R(\mathbf{1})$. Recall from Section 1.2 that we have for all $X$ in $\mathcal{C}: R F X \simeq A \otimes X \simeq X \otimes A$. In particular, the left and right cosets with respect to $\mathcal{A}=\langle A\rangle$ coincide.

On the other hand, for $X, Y \in \Lambda_{\mathcal{C}}$ we have

$$
\begin{gathered}
X \sim^{F} Y \Longleftrightarrow \operatorname{Hom}_{\mathcal{D}}(F Y, F X) \neq 0 \Longleftrightarrow \operatorname{Hom}_{C}(Y, R F X) \neq 0 \\
\Longleftrightarrow \operatorname{Hom}_{\mathcal{C}}(Y, A \otimes X) \neq 0 .
\end{gathered}
$$

In particular if $X \sim^{F} Y, Y$ is a factor of $A \otimes X$, so $X r_{\mathcal{A}}^{\mathcal{C}, r} Y$. By transitivity, $X \approx^{F} Y \Rightarrow X r_{\mathcal{A}}^{\mathcal{C}, r} Y$.

Conversely, assume $X r_{\mathcal{A}}^{\mathcal{C}, r} Y$. That means that there exists $Z \in \Lambda_{\mathcal{A}}$ such that $Y$ is a factor of $Z \otimes X$. Now the simple objects of $\mathcal{A}$ are the simple factors of $A^{\otimes n}$ and their duals. If $Z$ is a factor of $A^{\otimes n}$ with $n \geq 0$, one verifies by induction on $n$ that $X \approx^{F} Y$. The case where $Z$ is a dual of a factor of $A^{\otimes n}$ reduces to the previous case, since $X$ is a factor of $Z^{*} \otimes Y$. Thus $X r_{\mathcal{A}}^{\mathcal{C}, r} Y \Rightarrow X \approx^{F} Y$. 
3.2. An equivalence relation on $\Lambda_{\mathcal{D}}$ induced by $F$. Similarly, define a relation $\sim_{F}$ on $\Lambda_{\mathcal{D}}$ by $Y \sim_{F} Y^{\prime} \Longleftrightarrow Y_{F} \cap Y_{F}^{\prime} \neq \emptyset$. In other words, $Y \sim_{F} Y^{\prime}$ if there exists $X \in \Lambda_{\mathcal{C}}$ such that $F(X)$ contains both $Y$ and $Y^{\prime}$ as factors. One observes immediately that $\sim_{F}$ is symmetric, and it is reflexive if and only if $F$ is dominant. In general $\sim_{F}$ is not transitive.

If $F$ is dominant, we denote by $\approx_{F}$ the transitive closure of $\sim_{F}$, which is an equivalence relation on $\Lambda_{\mathcal{D}}$.

Example 3.4. Consider $K$ a Hopf subalgebra of a semisimple Hopf algebra $H$ and let $F$ be the restriction functor $F: \operatorname{Rep}(H) \rightarrow \operatorname{Rep}(K)$. Then in the paper [5], the equivalence relation $\approx^{F}$ was denoted by $u_{K}^{H}$ and the equivalence relation $\approx_{F}$ by $d_{K}^{H}$. As explained in [5] these equivalence relations are similar to the equivalence relations introduced by Rieffel in [14]. They arise from the restriction functor attached to an arbitrary extension of semisimple rings in [14].

Proposition 3.5. Let $F: \mathcal{C} \rightarrow \mathcal{D}$ be a dominant tensor functor between fusion categories. Denote by $A_{1}, \ldots, A_{l}$ (resp. $\left.B_{1}, \ldots, B_{l^{\prime}}\right)$ the equivalent classes of the relation $\approx^{F}\left(\right.$ resp. $\left.\approx_{F}\right)$ on on $\Lambda_{\mathcal{C}}\left(\right.$ resp. $\left.\Lambda_{\mathcal{D}}\right)$.

Then $l=l^{\prime}$, and after reindexing the $B_{j}$ 's we have

$$
F_{!}\left(\mathrm{R}_{A_{i}}\right)=[\mathcal{C}: \mathcal{D}] \mathrm{R}_{B_{i}} \text { and } R_{!}\left(\mathrm{R}_{B_{i}}\right)=\mathrm{R}_{A_{i}} .
$$

for $1 \leq i \leq l$, where $[\mathcal{C}: \mathcal{D}]=\frac{\operatorname{FPdim}(C)}{\operatorname{FPdim}(D)}$.

Proof. The proposition results from the following two lemmas.

Lemma 3.6. Let $F: \mathcal{C} \rightarrow \mathcal{D}$ be a dominant tensor functor between fusion categories. Then:

$$
F_{!}\left(\mathrm{R}_{\mathcal{C}}\right)=[\mathcal{C}: \mathcal{D}] \mathrm{R}_{\mathcal{D}} \quad \text { and } \quad R_{!}\left(\mathrm{R}_{\mathcal{D}}\right)=\mathrm{R}_{\mathcal{C}}
$$

Proof. The first identity is proved in [9]. Now

$$
\begin{aligned}
R_{!}\left(\mathrm{R}_{\mathcal{D}}\right) & =\sum_{Y \in \Lambda_{\mathcal{D}}} \operatorname{FPdim}(Y) R[Y]=\sum_{X \in \Lambda_{\mathcal{C}}} \sum_{Y \in \Lambda_{\mathcal{D}}} \operatorname{FPdim}(Y) m_{\mathcal{C}}(X, R Y)[X] \\
& =\sum_{X \in \Lambda_{\mathcal{C}}} \sum_{Y \in \Lambda_{\mathcal{D}}} \operatorname{FPdim}(Y) m_{\mathcal{C}}(F X, Y)[X] \\
& =\sum_{X \in \Lambda_{\mathcal{C}}} \operatorname{FPdim}(F X)[X]=\sum_{X \in \Lambda_{\mathcal{C}}} \operatorname{FPdim}(X)[X]=\mathrm{R}_{\mathcal{C}}
\end{aligned}
$$

Lemma 3.7. Let $F: \mathcal{C} \rightarrow \mathcal{D}$ be a dominant tensor functor between fusion categories. Let $X, X^{\prime} \in \Lambda_{\mathcal{C}}$ and $Y, Y^{\prime} \in \Lambda_{\mathcal{D}}$ be such that $Y$ is a 
factor of $F(X)$ and $Y^{\prime}$ is a factor of $F\left(X^{\prime}\right)$. Then

$$
X \approx^{F} X^{\prime} \Longleftrightarrow Y \approx_{F} Y^{\prime}
$$

As a result, $F$ induces a bijection $\Lambda_{\mathcal{C}} / \approx^{F} \rightarrow \Lambda_{\mathcal{D}} / \approx_{F}$, defined by $A \mapsto A^{F}$, its inverse being defined by $B \mapsto B_{F}$.

Proof. The first assertion results immediately from the definition of $\approx^{F}$ and $\approx_{F}$, and the second is a direct consequence of the first and the fact that $F$ is dominant.

Now let us prove the Proposition. The second lemma shows that $l=l^{\prime}$, and we renumber the $B_{j}$ 's so that $B_{i}=A_{i}{ }^{F}$. Then the support of $F\left(\mathrm{R}_{A_{i}}\right)$ is $B_{i}$, and the support of $R\left(\mathrm{R}_{B_{i}}\right)$ is $A_{i}$. The Proposition now results from the first Lemma, by restricting the linear maps induced by $F$ and $R$ to the blocks corresponding with the equivalent classes of $\approx^{F}$ and $\approx_{F}$.

Remark 3.8. Observe that if $F$ is not dominant, one can still apply Proposition 3.5 to the dominant tensor functor $\mathcal{C} \rightarrow \mathcal{D}^{\prime}, X \mapsto F(X)$, where $\mathcal{D}^{\prime} \subset \mathcal{D}$ is the dominant image of $F$.

\section{Normal TENSOR FUnCTORS}

In this section we study the previous equivalence relations when $F$ is normal (in the sense of [3]). Recall that a tensor functor $F: \mathcal{C} \rightarrow \mathcal{D}$ between fusion categories is normal if for any simple object $X$ of $\mathcal{C}$, $m_{\mathcal{D}}(\mathbf{1}, F(X))>0 \Rightarrow F(X)$ is trivial.

Remark 4.1. In other words, $F$ is normal if and only if $\left(\left(\mathbf{1}_{\mathcal{D}}\right)_{F}\right)^{F}=$ $\left\{\mathbf{1}_{\mathcal{D}}\right\}$.

Recall that $\mathfrak{K e r}_{F}$ is the fusion subcategory of all objects $X$ of $\mathcal{C}$ such that $F(X)$ is trivial (that is, $F(X)=\mathbf{1}^{\mathrm{FPdim}(X)}$ ).

Theorem 4.2. If $F: \mathcal{C} \rightarrow \mathcal{D}$ is normal, then $\sim^{F}$ is an equivalence relation on $\Lambda_{\mathcal{C}}$ and coincides with $r_{\operatorname{ker}_{F}}^{\mathcal{C}, l}$ and $r_{\operatorname{ker}_{F}}^{\mathcal{C}, r}$.

If in addition $F$ is dominant, $\sim_{F}$ is an equivalence relation on $\Lambda_{\mathcal{D}}$.

Proof. Let $X, X^{\prime} \in \Lambda_{\mathcal{C}}$. If $X \sim^{F} X^{\prime}$, then $F(X)$ and $F\left(X^{\prime}\right)$ have a common simple factor $Y$. Consequently, $F\left(X^{\prime} \otimes X^{*}\right)$ contains $Y \otimes$ $Y^{*}$ which in turn contains 1. Now $m_{\mathcal{D}}\left(F\left(X^{\prime} \otimes X^{*}\right), \mathbf{1}\right)=m_{\mathcal{C}}\left(X^{\prime} \otimes\right.$ $\left.X^{*}, R \mathbf{1}\right)=m_{\mathcal{C}}\left(X^{\prime}, R \mathbf{1} \otimes X\right)$ so $X^{\prime}$ is a factor of $R \mathbf{1} \otimes X$. Since $F$ is normal, $R \mathbf{1}$ is an object of $\mathfrak{K e r}_{F}$ so $X r_{\operatorname{ker}_{F}}^{\mathcal{C}, l} X^{\prime}$.

On the other hand, if $X r_{\operatorname{ker}_{F}}^{\mathcal{C}, l} X^{\prime}$, there exists $E$ in $\mathfrak{K e r}_{F}$ such that $X^{\prime}$ is a factor of $E \otimes X$. Since $F(E) \simeq \mathbf{1}^{n}, F\left(X^{\prime}\right)$ is a factor of $F(X)^{n}$. This proves that $X^{\prime F} \subset X^{F}$, so $X \sim^{F} X^{\prime}$. 
This proves that $\sim^{F}$ is equal to $r_{\operatorname{ker}_{F}}^{\mathcal{C}, l}$; it is also equal to $r_{\operatorname{ker}_{F}}^{\mathcal{C}, r}$ by reason of symmetry, and to $\approx^{F}$ because it is already an equivalence relation.

Now assume $F$ is dominant. Let $Y, Y^{\prime} \in \Lambda_{\mathcal{D}}$ such that $Y \approx_{F} Y^{\prime}$. Let $X, X^{\prime} \in \Lambda_{\mathcal{C}}$ such that $Y \in X^{F}$ and $Y^{\prime} \in X^{\prime F}$. Then we have $X \approx^{F} X^{\prime}$ by Lemma 3.7, so $X r_{\operatorname{ker}_{F}}^{\mathcal{C}, l} X^{\prime}$. We have just seen that this implies $X^{\prime F} \subset X^{F}$, so $Y, Y^{\prime} \in X^{F}$, hence $Y \sim_{F} Y^{\prime}$. This proves that $\sim_{F}=\approx_{F}$ is an equivalence relation.

Corollary 4.3. Let $F: \mathcal{C} \rightarrow \mathcal{D}$ be a tensor functor between fusion categories and let $\mathcal{D}^{\prime}$ be its dominant image. The following assertions are equivalent:

(i) $F$ is normal;

(ii) for $X, X^{\prime} \in \Lambda_{\mathcal{C}}, X^{F}$ and $X^{\prime F}$ are either disjoint or equal;

(iii) for $Y, Y^{\prime} \in \Lambda_{\mathcal{D}^{\prime}}, Y_{F}$ and $Y_{F}^{\prime}$ are either disjoint or equal;

Proof. We may assume $F$ dominant and therefore $\mathcal{D}=\mathcal{D}^{\prime}$.

(ii) $\Longrightarrow\left(\right.$ i) : let $X \in \Lambda_{\mathcal{C}}$ such that $\mathbf{1}_{\mathcal{D}} \in X^{F}$. Since $\mathbf{1}_{\mathcal{C}}^{F}=\left\{\mathbf{1}_{\mathcal{D}}\right\}$, (ii) implies that $X^{F}=\left\{\mathbf{1}_{\mathcal{D}}\right\}$, that is $F(X)$ is trivial. Thus, $F$ is normal.

(iii) $\Longrightarrow$ (i) : let $X \in \Lambda_{\mathcal{C}}$ such that $\mathbf{1}_{\mathcal{D}} \in X^{F}$, and let $Y \in X^{F}$. We have $X \in\left(\mathbf{1}_{\mathcal{D}}\right)_{F} \cap Y_{F}$, so (iii) implies $Y_{F}=\left(\mathbf{1}_{\mathcal{D}}\right)_{F}$. In particular $\mathbf{1}_{\mathcal{C}} \in Y_{F}$, which means $Y=\mathbf{1}_{\mathcal{D}}$. This shows that $F(X)$ is trivial. Thus, $F$ is normal.

(i) $\Longrightarrow$ (ii) and (iii) because if $F$ is normal, by Theorem 4.2 the $X^{F}$ 's and the $Y_{F}$ 's are equivalence classes for $\sim^{F}\left(\right.$ resp. $\left.\sim_{F}\right)$.

The above Corollary can be regarded as an analogue of the fact that a Hopf subalgebra is depth two if and only if it is normal. See [5] for a proof in the context of Hopf algebras.

Theorem 4.4. Let $F: \mathcal{C} \rightarrow \mathcal{D}$ be a normal tensor functor between fusion categories, and denote by $R$ its right adjoint. Let $A_{1}, \ldots, A_{l}$ denote the equivalence classes of $\approx^{F}$ in $\Lambda_{\mathcal{C}}$,

(1) For $X \in A_{i}$

$$
\frac{F_{!}[X]}{\operatorname{FPdim}(X)}=\frac{F_{!}\left(\mathrm{R}_{A_{i}}\right)}{\operatorname{FPdim}\left(\mathrm{R}_{A_{i}}\right)},
$$

(2) for $X, X^{\prime} \in \Lambda_{\mathcal{C}}$ :

$$
X \approx^{F} X^{\prime} \Longleftrightarrow \frac{F ![X]}{\operatorname{FPdim}(X)}=\frac{F_{!}\left[X^{\prime}\right]}{\operatorname{FPdim}\left(X^{\prime}\right)}
$$

Now assume in addition that $F$ is dominant, and let $B_{1}, \ldots, B_{l}$ be the equivalence classes of $\approx_{F}$ in $\Lambda_{\mathcal{D}}$, with $B_{i}=\left(A_{i}\right)_{F}$. Then: 
(3) for $Y \in B_{i}$,

$$
\frac{R_{!}[Y]}{\operatorname{FPdim}(Y)}=[\mathcal{C}: \mathcal{D}] \frac{\mathrm{R}_{A_{i}}}{\operatorname{FPdim}\left(\mathrm{R}_{A_{i}}\right)},
$$

(4) for $Y, Y^{\prime} \in \Lambda_{\mathcal{D}}$ :

$$
Y \approx_{F} Y^{\prime} \Longleftrightarrow \frac{R_{!}[Y]}{\operatorname{FPdim}(Y)}=\frac{R_{!}\left[Y^{\prime}\right]}{\operatorname{FPdim}\left(Y^{\prime}\right)} .
$$

Proof. 1) Let $X \in A_{i}$. By Theorem 4.2, the $A_{i}$ 's are the left cosets relative to the fusion subcategory $\mathfrak{K e r}_{F}$. In particular, the class of $\mathbf{1}$ is $\mathfrak{K e r}_{F}$. Relation (2.1) implies that

$$
\mathrm{R}_{\mathfrak{K e r}_{F}}[X]=\operatorname{FPdim}(X) \frac{\operatorname{FPdim}\left(\mathfrak{K e r}_{F}\right)}{\operatorname{FPdim}\left(\mathrm{R}_{A_{i}}\right)} \mathrm{R}_{\mathcal{A}_{i}} .
$$

Applying $F_{!}$, and noting that $F_{!}\left(\mathrm{R}_{\mathfrak{K e r}_{F}}\right)=\operatorname{FPdim}\left(\mathfrak{K e r}_{F}\right)[\mathbf{1}]$, we obtain the formula of Assertion (1).

2) An immediate consequence of the first assertion is that

$$
X \approx^{F} X^{\prime} \Rightarrow \operatorname{FPdim}(X)^{-1} F_{!}[X]=\operatorname{FPdim}\left(X^{\prime}\right)^{-1} F_{!}\left[X^{\prime}\right] .
$$

On the other hand, if $X, X^{\prime} \in \Lambda_{\mathcal{C}}$ are such that $F_{!}[X]$ and $F_{!}\left[X^{\prime}\right]$ are colinear in $K_{0}(\mathcal{D})_{\mathbb{C}}$, then $F(X)$ and $F\left(X^{\prime}\right)$ have a common simple factor so $X \sim^{F} X^{\prime}$. This proves Assertion (2).

3) Let $Y \in B_{i}$. We have $Y^{F}=A_{i}$, and

$$
\begin{aligned}
R_{!}[Y] & =\sum_{X \in \Lambda_{\mathcal{C}}} m_{\mathcal{C}}(X, R(Y))[X]=\sum_{X \in \Lambda_{\mathcal{C}}} m_{\mathcal{D}}(F(X), Y)[X] \\
& =\sum_{X \in A_{i}} m_{\mathcal{D}}(F(X), Y)[X] \\
& =\sum_{X \in A_{i}} m_{\mathcal{D}}\left(\frac{F(X)}{\operatorname{FPdim}(X)}, Y\right) \operatorname{FPdim}(X) X \\
& =m_{\mathcal{D}}\left(Y, \frac{F\left(\mathrm{R}_{A_{i}}\right)}{\operatorname{FPdim}\left(A_{i}\right)}\right) \sum_{X \in A_{i}} \operatorname{FPdim}(X) X \\
& =m_{\mathcal{D}}\left(Y, \frac{[\mathcal{C}: \mathcal{D}] \mathrm{R}_{B_{i}}}{\operatorname{FPdim}\left(A_{i}\right)}\right) \sum_{X \in A_{i}} \operatorname{FPdim}(X) X \\
& =[\mathcal{C}: \mathcal{D}] \operatorname{FPdim}(Y) \frac{\mathrm{R}_{A_{i}}}{\operatorname{FPdim}\left(A_{i}\right)}
\end{aligned}
$$

hence Assertion (3).

4) Assertion (3) shows that

$$
Y \approx_{F} Y^{\prime} \Rightarrow \operatorname{FPdim}(Y)^{-1} R_{!}[Y]=\operatorname{FPdim}\left(Y^{\prime}\right)^{-1} R_{!}\left[Y^{\prime}\right] .
$$


On the other hand if $R_{!}[Y]$ and $R_{!}\left[Y^{\prime}\right]$ are colinear virtual objects, then $R(Y)$ and $R\left(Y^{\prime}\right)$ have a common simple factor so $Y \sim_{F} Y^{\prime}$. This proves Assertion (4).

Recall [3] that a fusion subcategory $\mathcal{D} \subset \mathcal{C}$ is called normal if there is a normal tensor functor $F: \mathcal{C} \rightarrow \mathcal{E}$ such that $\mathcal{D}=\mathfrak{K e r}_{F}$.

The following Proposition, which is a straightforward consequence of Theorem 4.2 and 2.1, can be seen as a generalization of the fact that left and right cosets of a normal subgroup coincide. Its analogue for Hopf algebras was proven in [4].

Corollary 4.5. If $\mathcal{D}$ is a normal fusion subcategory of $\mathcal{C}$ then the left and right cosets of $\mathcal{C}$ relative to $\mathcal{D}$ coincide. Moreover, $\mathrm{R}_{\mathcal{D}}$ is then central in $K_{0}(\mathcal{C})_{\mathbb{C}}$.

\section{Normality AND COMPOSITION OF FUnCTORS, With AN APPLICATION TO EQUIVARIANTIZATIONS}

In this section, we study the following question: if the composition of two tensor functors is normal, what can be said about each functor? We apply our result to the special case of equivariantizations.

Theorem 5.1. Consider a commutative triangle of tensor functors between fusion categories:

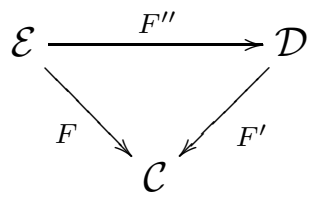

and assume that $F$ is normal and $F^{\prime \prime}$ is dominant. Then

(1) The functor $F^{\prime}$ is normal;

(2) Denoting by $\mathbb{A}$ and $\mathbb{A}^{\prime \prime}$ the central induced algebra of $F$ and $F^{\prime \prime}$, which are commutative algebras in the categorical center $\mathcal{Z}(\mathcal{C})$ of $\mathcal{C}, \mathbb{A}^{\prime \prime}$ is a subalgebra of $\mathbb{A}$.

(3) The functor $F^{\prime \prime}$ induces a dominant tensor functor

$$
F_{0}^{\prime \prime}: \mathfrak{K e r}_{F} \rightarrow \mathfrak{K e r}_{F^{\prime}},
$$

with $\mathfrak{K e r}_{F_{0}^{\prime \prime}}=\mathfrak{K e r}_{F^{\prime \prime}}$, and the following assertions are equivalent:

(i) $F^{\prime \prime}$ is normal;

(ii) $F_{0}^{\prime \prime}$ is normal;

(iii) $\operatorname{FPdim}\left(\mathfrak{K e r}_{F^{\prime \prime}}\right)=\left[\mathfrak{K e r}_{F}: \mathfrak{K e r}_{F^{\prime}}\right]$.

Remark 5.2. The first assertion of Theorem 5.1 generalizes the classical fact that if a subgroup is normal, then it is normal in any intermediate subgroup. 
Remark 5.3. Denote by $H$ and $K$ the induced Hopf algebras of the normal functors $F$ and $F^{\prime}$ respectively, so that we may identify $\mathfrak{K e r}_{F}$ to $\operatorname{comod}(H)$ and $\mathfrak{K e r}_{F^{\prime}}$ to $\operatorname{comod}(K)$; then the dominant tensor functor $F_{0}^{\prime \prime}$ is induced by a surjective Hopf algebra morphism $p: H \rightarrow K$, and condition (ii) of Assertion (3) of the Theorem means that $K^{*}$ is a normal Hopf subalgebra of $H^{*}$ (see [3] ).

Proof. Assertion (1). Let $Y \in \Lambda_{\mathcal{D}}$ and assume that $F^{\prime}(Y)$ contains 1. Since $F^{\prime \prime}$ is dominant, there exists $X \in \Lambda_{\mathcal{E}}$ such that $F^{\prime \prime}(X)$ contains $Y$. Therefore, $F^{\prime} F^{\prime \prime}(X)=F(X)$ contains $\mathbf{1}$, and since $F$ is normal, $F(X)$ is trivial. So $F^{\prime}(Y)$, being contained in $F^{\prime} F^{\prime \prime}(X)$, is trivial too. This shows that $F^{\prime}$ is normal.

Assertion (3). Clearly, $F^{\prime \prime}\left(\mathfrak{K e r}_{F}\right) \subset \mathfrak{K e r}_{F^{\prime}}$; so $F^{\prime \prime}$ induces by restriction a tensor functor $F_{0}^{\prime \prime}: \mathfrak{K e r}_{F} \rightarrow \mathfrak{K e r}_{F^{\prime}}$. The kernel of $F_{0}^{\prime \prime}$ is $\mathfrak{K e r}_{F^{\prime \prime}}$.

Key fact: if $X \in \Lambda_{\mathcal{E}}$ is such that $F^{\prime \prime}(X)$ contains $Y \in \Lambda_{\mathfrak{K e r}_{F^{\prime}}}$, then $X$ belongs to $\mathfrak{K e r}_{F}$. Indeed, in that case $F(X)=F^{\prime} F^{\prime \prime}(X)$ contains $F^{\prime}(Y) \simeq \mathbf{1}^{\mathrm{FPdim}(Y)}$, so, $F$ being normal, $F(X)$ is trivial.

From the key fact, we draw two consequences:

1) If $X \in \Lambda_{\mathcal{E}}$ is such that $F^{\prime \prime}(X)$ contains $\mathbf{1}$, then $X \in \mathfrak{K e r}_{F}$. This shows that $F^{\prime \prime}$ is normal if and only if $F_{0}^{\prime \prime}$ is normal, thus (i) $\Longleftrightarrow$ (ii).

2) The functor $F_{0}^{\prime \prime}$ is dominant. Indeed, for $Y \in \Lambda_{\mathfrak{K e r}_{F^{\prime}}}$, there exists $X \in \Lambda_{\mathcal{C}}$ such that $F(X)$ contains $Y$, because $F^{\prime \prime}$ is dominant; and the key fact insures that $X \in \mathfrak{K e r}_{F}$.

Denote by $i$ the inclusion $\mathfrak{K e r}_{F^{\prime \prime}} \hookrightarrow \mathfrak{K e r}_{F}$, and consider the sequence of tensor functors:

$$
\text { (E) } \mathfrak{K e r}_{F^{\prime \prime}} \stackrel{i}{\longrightarrow} \mathfrak{K e r}_{F} \stackrel{F_{0}^{\prime \prime}}{\longrightarrow} \mathfrak{K e r}_{F^{\prime}}
$$

where $F_{0}^{\prime \prime}$ is dominant and $\mathfrak{K e r}_{F^{\prime \prime}}$ is its kernel.

We may apply Proposition 1.1; we have

$$
\operatorname{FPdim}\left(\mathfrak{K} \mathfrak{r r}_{F}\right) \geq \operatorname{FPdim}\left(\mathfrak{K}_{\mathfrak{e r}} \mathfrak{F}_{F^{\prime}}\right) \operatorname{FPdim}\left(\mathfrak{K e r}_{F^{\prime \prime}}\right)
$$

and equality holds if and only if $(E)$ is an exact sequence, that is, $F_{0}^{\prime \prime}$ is normal. This shows (ii) $\Longleftrightarrow$ (iii).

Assertion (2). Recall that the induced central algebra $\mathbb{A}$ of $F$ is defined as follows. See [3] for details, and [1] for a more general account (with the dual point of view). Denote by $R^{\prime}, R^{\prime \prime}$ the right adjoints of $F^{\prime}$ and $F^{\prime \prime}$ respectively, so that $R=R^{\prime \prime} R^{\prime}$ is right adjoint to $F$. Let $\hat{T}=R F=R^{\prime \prime} R^{\prime} F^{\prime} F^{\prime \prime}$ be the monad of the Hopf monoidal adjunction $(F, R)$. Then $\hat{T}$ is a monoidal monad on $\mathcal{E}$. Let $A=\hat{T}(\mathbf{1})$. For $X$ an object of $\mathcal{E}$, define morphisms $u_{X}: A \otimes X \rightarrow \hat{T}(X)$ and $v_{X}: X \otimes A \rightarrow$ 
$\hat{T}(X)$ by the commutativity of the following diagrams:

$$
\begin{aligned}
& A \otimes X \underset{A \otimes \eta_{X}}{\longrightarrow} A \otimes \hat{T}(X) \underset{\hat{T}_{2}(\mathbf{1}, X)}{\longrightarrow} \hat{T}(X) \\
& X \otimes A \underset{\eta_{X} \otimes A}{\longrightarrow} \hat{T}(X) \otimes A \underset{\hat{T}_{2}(X, \mathbf{1})}{\longrightarrow} \hat{T}(X),
\end{aligned}
$$

where $\eta$ is the unit of the monad $\hat{T}$, and $\hat{T}_{2}$ is its monoidal structure. Then $u_{X}$ and $v_{X}$ are in fact isomorphisms (because the adjunction $(F, R)$ is Hopf). Define an isomorphism $\sigma: A \otimes \mathrm{id}_{\mathcal{E}} \rightarrow \mathrm{id}_{\mathcal{E}} \otimes A$ by

$$
\sigma_{X}=v_{X}^{-1} u_{X}
$$

Then $\sigma$ is a half-braiding in $\mathcal{E}$, and $\mathbb{A}=(A, \sigma)$.

Similarly, $\mathbb{A}^{\prime \prime}=\left(A^{\prime \prime}, \sigma^{\prime \prime}\right)$ is defined in terms of the monoidal monad $\hat{T}^{\prime \prime}=R^{\prime \prime} F^{\prime \prime}$ of the Hopf monoidal adjunction $\left(F^{\prime \prime}, R^{\prime \prime}\right)$.

Now the unit $\eta^{\prime}: 1_{\mathcal{D}} \rightarrow R^{\prime} F^{\prime}$ of the monoidal adjunction $\left(F^{\prime}, R^{\prime}\right)$ defines a natural transformation $f=R^{\prime \prime} \eta^{\prime} T^{\prime \prime}: \hat{T}^{\prime \prime} \rightarrow \hat{T}$ which is clearly a monoidal morphism of monads. In particular, $f_{\mathbf{1}}$ is an algebra morphism $A^{\prime \prime} \rightarrow A$. Moreover, since $\sigma$ and $\sigma^{\prime \prime}$ are defined in terms of the monoidal monad structures of $\hat{T}$ and $\hat{T}^{\prime \prime}$, which are preserved by $f$, one verifies easily that $\left(X \otimes f_{\mathbf{1}}\right) \sigma_{X}^{\prime \prime}=\sigma_{X}\left(f_{\mathbf{1}} \otimes X\right)$, that is, $f_{\mathbf{1}}$ is an algebra morphism from $\mathbb{A}^{\prime \prime}$ to $\mathbb{A}$ in $\mathcal{Z}(\mathcal{C})$.

Lastly, $f$ is a monomorphism because $F^{\prime}$ being faithful, $\eta^{\prime}: \mathrm{id}_{\mathcal{D}} \rightarrow$ $R^{\prime} F^{\prime}$ is a monomorphism and $R^{\prime \prime}$ preserves monomorphisms. In particular, $\mathbb{A}^{\prime \prime}$ is a subalgebra of $\mathbb{A}$ via $f_{\mathbf{1}}$.

We can apply Theorem 5.1 to equivariantizations (see Section 1.3).

Corollary 5.4. Let $G$ be a finite group acting on a fusion category $\mathcal{C}$ by autoequivalences. Assume that the base field $\mathbb{k}$ is algebraically closed, and its characteristic does not divide the order $|G|$ of $G$, so that the equivariantization $\mathcal{C}^{G}$ is a fusion category. Let $H$ be a subgroup of $G$. Then the restriction functor $r_{H}^{G}: \mathcal{C}^{G} \rightarrow \mathcal{C}^{H}$ is normal if and only if $H$ is a normal subgroup of $G$.

Moreover, in that case the group $G / H$ acts on $\mathcal{C}^{H}$ by tensor autoequivalences, and $\mathcal{C}^{G}$ is tensor equivalent to $\left(\mathcal{C}^{H}\right)^{G / H}$ in such a way that the following diagram of tensor functors commutes:

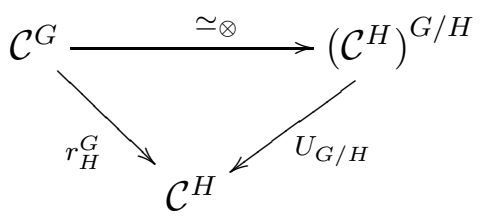


Proof. We apply Theorem 5.1 to $\mathcal{C}=\mathcal{C}, \mathcal{D}=\mathcal{C}^{H}, \mathcal{E}=\mathcal{C}^{G}, F: \mathcal{C}^{G} \rightarrow \mathcal{C}$, $F^{\prime}: \mathcal{C}^{H} \rightarrow \mathcal{C}$ are the forgetful functors, and $F^{\prime \prime}=r_{H}^{G}: \mathcal{C}^{G} \rightarrow \mathcal{C}^{H}$ is the restriction functor defined by sending an object $\left(X,\left(r_{g}\right)_{g \in G}\right)$ of $\mathcal{C}^{G}$ to the object $\left(X,\left(r_{h}\right)_{h \in H}\right)$ of $\mathcal{C}^{H}$.

In this case, ${\mathfrak{K} e \mathfrak{r}_{F}}=\operatorname{Rep}(G), \mathfrak{K e r}_{F^{\prime}}=\operatorname{Rep}(H)$, and the functor

$$
F_{0}^{\prime \prime}: \mathfrak{K e r}_{F} \rightarrow \mathfrak{K}_{\mathfrak{K} \mathfrak{r}_{F}^{\prime \prime}}
$$

is just the restriction functor $\operatorname{Rep}(G) \rightarrow \operatorname{Rep}(H)$. Thus $\mathfrak{K e r}_{F_{0}^{\prime \prime}}=$ $\mathfrak{K e r}_{F^{\prime \prime}}=\operatorname{Rep}(G / \bar{H})$, where $\bar{H}$ is the normal subgroup of $G$ generated by $H$. The equivalence of (i) and (iii) in Assertion (3) tells us that $F^{\prime \prime}$ is normal if and only if $\mathrm{FPdim}(\operatorname{Rep}(G / \bar{H}))=\frac{\mathrm{FPdim}(\operatorname{Rep}(G))}{\operatorname{FPdim}(\operatorname{Rep}(H))}$, that is, $|G / \bar{H}|=|G / H|$, which is equivalent to $\bar{H}=H$, in other words $H$ is normal in $G$.

Now assume $H$ is normal in $G$. In addition to the exact sequence:

$$
\text { (E) } \mathfrak{K}_{\mathfrak{i}} \mathfrak{r}_{F}=\operatorname{Rep}(G) \longrightarrow \mathcal{C}^{G} \stackrel{F}{\longrightarrow} \mathcal{C},
$$

we have a second exact sequence of fusion categories:

$$
\left(E^{\prime \prime}\right) \quad \mathfrak{K e r}_{F^{\prime \prime}}=\operatorname{Rep}(G / H) \longrightarrow \mathcal{C}^{G} \stackrel{F^{\prime \prime}}{\longrightarrow} \mathcal{C}^{H} .
$$

The induced Hopf algebra of $(E)$ is $\mathbb{k}^{G}$, and that of $\left(E^{\prime \prime}\right)$ is $\mathbb{k}^{G / H}$. According to the equivariantization criterium of Theorem 1.2, $(E)$ is a central exact sequence. Let $\mathbb{A}=(A, \sigma)$ and $\mathbb{A}^{\prime \prime}=\left(A^{\prime \prime}, \sigma^{\prime \prime}\right)$ denote the central induced algebras of $F$ and $F^{\prime \prime}$ respectively. Centrality of $(E)$ means that the forgetful functor $\langle\mathbb{A}\rangle \rightarrow\langle A\rangle$ is an equivalence of categories. By Assertion (2) of Theorem 5.1, $\mathbb{A}^{\prime}$ is a subalgebra of $\mathbb{A}$, so that $\left\langle\mathbb{A}^{\prime \prime}\right\rangle$ is a fusion subcategory of $\langle\mathbb{A}\rangle$. The forgetful functor $\left\langle\mathbb{A}^{\prime \prime}\right\rangle \rightarrow\left\langle A^{\prime \prime}\right\rangle$ is full and dominant, so it is an equivalence, which means that $\left(E^{\prime \prime}\right)$ is central. Now, again by Theorem 1.2, $\left(E^{\prime \prime}\right)$ is an equivariantization exact sequence, with group $G / H$, and we are done.

\section{ON THE RADICAL AND COMMUTATOR OF A NORMAL FUSION SUBCATEGORY}

In this section we introduce the radical of a fusion subcategory and compare it to the commutator in the case of a normal fusion subcategory.

Let $\mathcal{C}$ be a fusion category, let and $\mathcal{D}$ be a fusion subcategory of $\mathcal{C}$.

The radical of $\mathcal{D}$ in $\mathcal{C}$, denoted by $\operatorname{rad}_{\mathcal{C}}(\mathcal{D})$, is the full abelian subcategory of $\mathcal{C}$ generated by the simple objects $X$ of $\mathcal{C}$ such that $X^{\otimes n}$ belongs to $\mathcal{D}$ for some integer $n>0$. 
The commutator of $\mathcal{D}$ in $\mathcal{C}$, denoted by $\mathcal{D}^{c o}$, is the full abelian subcategory of $\mathcal{C}$ generated by the simple objects $X$ of $\mathcal{C}$ such that $X \otimes X^{*}$ belongs to $\mathcal{D}$. This notion is introduced in [11.

Note that if $K_{0}(\mathcal{C})$ is commutative (for example $\mathcal{C}$ braided) then $\mathcal{D}^{c o}$ and $\operatorname{rad}_{\mathcal{C}}(\mathcal{D})$ are fusion subcategories of $\mathcal{C}$, but not in general.

For a normal fusion subcategory the radical and the commutator coincide. Indeed:

Proposition 6.1. Let $\mathcal{C}$ be a fusion category, and let $\mathcal{D} \subset \mathcal{C}$ be a normal fusion subcategory of $\mathcal{C}$. Denote by $F: \mathcal{C} \rightarrow \tilde{\mathcal{C}}$ a tensor functor between fusion categories such that $\mathcal{D}=\mathfrak{K e r}_{F}$.

Let $\mathcal{E}$ be the full abelian subcategory of $\mathcal{C}$ generated by the simple objects $X$ of $\mathcal{C}$ such that $F(X)=M^{n}$, with $M$ invertible and $n \geq 0$. Then $\mathcal{D}^{c o}=\operatorname{rad}_{\mathcal{C}}(\mathcal{D})=\mathcal{E}$. In particular, $\mathcal{D}^{\text {co }}$ and $\operatorname{rad}_{\mathcal{C}}(\mathcal{D})$ are fusion subcategories of $\mathcal{C}$.

Proof. Observe that $\mathcal{E}$ is a fusion subcategory, because clearly a simple factor of the tensor product of two simple objects of $\mathcal{E}, \mathbf{1}$, and the dual of a simple object of $\mathcal{E}$, all belong to $\mathcal{E}$.

In order to show the inclusion $\mathcal{D}^{c o} \subset \mathcal{E}$, we will need the following

Lemma 6.2. Let $\mathcal{C}$ be a fusion category, and $X \in \Lambda_{\mathcal{C}}$. The following assertions are equivalent:

(i) $X$ is a multiple of an invertible object of $\mathcal{C}$;

(ii) There exists $n>0$ such that $X^{\otimes n}$ is trivial.

Proof. (i) $\Longrightarrow$ (ii) because $\operatorname{Inv}(\mathcal{C})$ is a finite group, so invertible objects of $\mathcal{C}$ have finite order. (ii) $\Longrightarrow$ (i). Assume $X^{\otimes n}$ is trivial for some $n>0$, and let us show that $X$ is a multiple of an invertible object. We begin with the case where $X$ is simple. Since $\mathbf{1}$ is a factor of $X \otimes X^{\otimes n-1}, X^{*}$ is a factor of $X^{\otimes n-1}, X \otimes X^{*}$ is a factor of $X^{\otimes n}$ and in particular $X \otimes X^{*}$ is trivial. Now $m_{\mathcal{C}}\left(\mathbf{1}, X \otimes X^{*}\right)=m_{\mathcal{C}}(X, X)=1$, so $X \otimes X^{*}=\mathbf{1}$, that is, $X$ is invertible. Now for the general case. Let $M$ be a simple factor of $X$. Since $X^{\otimes n}$ contains $M^{\otimes n}, M$ is invertible. Let $N$ be another simple (also, invertible) factor of $X$. Then $M^{\otimes n}$ contains $M^{\otimes n}$ and $N \otimes M^{\otimes n-1}$, which are both trivial and simple and therefore isomorphic to 1 . This implies $M \simeq N$, so $X$ is a multiple of $M$.

Now let $X$ be a simple object of $\operatorname{rad}_{\mathcal{C}}(\mathcal{D})$. There exists $n>0$ such that $F(X)^{\otimes n}$ is trivial, and by the Lemma, $F(X)$ is a multiple of an invertible object of $\tilde{\mathcal{C}}$, so $X$ belongs to $\mathcal{E}$. $\operatorname{Thus} \operatorname{rad}_{\mathcal{C}}(\mathcal{D}) \subset \mathcal{E}$.

Now if $X$ be a simple object of $\mathcal{D}^{c o}$, then $F(X) \otimes F(X)^{*}$ is trivial. If $M, N$ are two simple factors of $F(X), M \otimes N^{*}$ is trivial, which implies that $M=N$ is invertible, and $X$ lies in $\Lambda_{\mathcal{E}}$. Thus $\mathcal{D}^{c o} \subset \mathcal{E}$. 
Conversely, let $X$ be a simple object of $\mathcal{E}$. We have $F(X)=M^{m}$, with $M$ invertible. By the lemma, there exists $n>0$ such that $F(X)^{\otimes m}$ is trivial, so $X^{\otimes m}$ belongs to $\mathcal{D}$, and $X$ belongs to $\operatorname{rad}_{\mathcal{C}}(\mathcal{D})$. Also, $F\left(X \otimes X^{*}\right)$ is trivial, so $X \otimes X^{*}$ belongs to $\mathcal{D}$, and $X$ belongs to $\mathcal{D}^{\text {co }}$.

Thus, $\operatorname{rad}_{\mathcal{C}}(\mathcal{D})=\mathcal{D}^{c o}=\mathcal{E}$.

Acknowledgments. The work of Sebastian Burciu was supported by a grant of the Romanian National Authority for Scientific Research, CNCS - UEFISCDI, project number PN-II-RU-TE-2012-3-0168.

\section{REFERENCES}

1. A. Bruguières, S. Lack, and A. Virelizier, Hopf monads on monoidal categories, Adv. Math. 227 (2011), no. 2, 745-800.

2. A. Bruguières and S. Natale, Central Exact Sequences of Tensor Categories, Equivariantization and Applications, to appear in J. Math. Soc. Japan. Preprint arXiv:1112.3135.

3. $5644-5705$.

4. S. Burciu, Coset Decomposition For Semisimple Hopf Algebras, Comm. Alg. 10 (2009), 3573-3585.

5. S. Burciu, L. Kadison, and B. Külshammer, On subgroup depth, Int. Electr. J. Alg. 9 (2011), 133-166.

6. S. Burciu and S. Natale, Fusion rules of equivariantizations of fusion categories, J. Math. Phys. 54 (2013), 013511.

7. P. Deligne, Categories tannakiennes, vol. 87, Progr. Math. 87, Birkhauser Boston, 111-195, The Grothendieck Festschrift, Vol. II., 1990.

8. V. Drinfeld, S. Gelaki, D. Nikshych, and V. Ostrik, On braided fusion categories, I, Sel. Math. New Ser. (2010), no. 16, 1-119.

9. P. Etingof, D. Nikshych, and V. Ostrik, On fusion categories, Annals of Mathematics 162 (2005), 581-642.

10. F.R. Gantmacher, Matrizentheorie, Springer, Berlin, 1986.

11. S. Gelaki and D. Nikshych, Nilpotent fusion categories, Adv. Math. 217 (2008), no. 3, 1053-1071.

12. D. Nikshych, Non group-theoretical semisimple Hopf algebras from group actions on fusion categories, Sel. Math. 14 (2009), no. 1, 145 - 161.

13. V Ostrik, Module categories, weak Hopf algebras and modular invariants, Transform. Groups 26 (2003), no. 8, 177-206.

14. M. Rieffel, Normal subrings and induced representations, J. Alg. 24 (1979), 264-386.

Département de Mathématiques, Université MontPellier II, 34095 Montpellier Cedex 5, France

E-mail address: bruguier@math.univ-montp2.fr, alain.bruguieres@gmail.com

and 
Simion Stoilow Institute of Mathematics of the Romanian Academy, Research Unit 5, P.O. Box 1-764, RO-014700, Bucharest, Romania

E-mail address: sebastian.burciu@imar.ro, smburciu@gmail.com 